\title{
RESPONSABILIDADES DOS INTERMEDIÁRIOS EM UMA REDE DE LOGÍSTICA REVERSA DE EQUIPAMENTOS MÉDICOS EM UM SISTEMA PRODUTO- SERVIÇO
}

Filipe Jané Alcarás (filipe.alcaras@usp.br) - Departamento de Engenharia de Produção, Escola de Engenharia de São Carlos, Universidade de São Paulo (USP).

Adriana Hofmann Trevisan (adrianatrevisan@usp.br) - Departamento de Engenharia de Produção, Escola de Engenharia de São Carlos, Universidade de São Paulo (USP).

Janaina Mascarenhas Hornos da Costa (janainacosta@usp.br) - Departamento de Engenharia de Produção, Escola de Engenharia de São Carlos, Universidade de São Paulo (USP).

\section{RESUMO}

Sistemas Produto-Serviço (PSSs) são soluções, com potencial para a economia circular, que satisfazem as necessidades dos clientes por meio da combinação de produtos e serviços. Nessa solução, geralmente o provedor do PSS permanece com a posse do artefato e, ao término da oferta, o produto é retornado à empresa através de redes de logística reversa (LR). No setor da saúde, pressões legislativas têm impulsionado as empresas médico-hospitalares a adotarem estruturas de LR para reprocessarem produtos usados ou para descartá-los adequadamente no fim de vida. Para auxiliar na prestação de serviços, incluindo atividades de fluxo reverso de materiais, os provedores de PSS podem trabalhar em conjunto com organizações intermediárias/distribuidoras, que são essenciais para a completude da solução. Assim, o objetivo dessa pesquisa é identificar as responsabilidades dos intermediários em uma rede de LR de equipamentos médicos. Para isto, foram realizadas entrevistas semiestruturadas com quatro empresas que auxiliam os fornecedores de PSS na prestação de serviços diversos para clientes de bombas de infusão. Os resultados indicam que a inexistência desses parceiros de negócio inviabilizaria atividades desde a coleta do produto até a disponibilização do mesmo para novos clientes.

Palavras chave: Sistema Produto-Serviço; Logística Reversa; Third Party Reverse Logistics Provider; Economia Circular, Dispositivos Médicos 


\section{INTRODUÇÃO}

Para solucionar problemas ambientais, muitas abordagens e conceitos de controle da poluição emergiram com o passar do tempo, como produção mais limpa, eco-design e design do ciclo de vida do produto (VEZZOLI et al., 2015). Embora essas abordagens sejam relevantes, Mont (2002) argumenta que é necessária uma nova estratégia para estimular a mudança nos padrões de produção e consumo atuais.

Uma opção emergente é através de Sistemas Produtos-Serviço (PSSs), que são soluções integradas de produtos com serviços (UNEP, 2002), projetadas para satisfazer as necessidades dos usuários durante todo o ciclo de vida do produto (SUNDIN; BRAS, 2005) e gerar menos impacto ambiental do que os tradicionais modelos de negócio (GOEDKOOP et al., 1999).

No PSS, a propriedade do produto geralmente permanece com o provedor da solução (BAINES et al., 2007; BEUREN; FERREIRA; MIGUEL, 2013). As empresas que integram serviços ao produto podem firmar contratos com os clientes para devolução do produto ao término da vida útil/uso (CHIERICI; COPANI, 2016). Porém, para que os produtos, peças e materiais (resíduos sólidos) consigam retornar ao setor empresarial, é necessária uma rede de logística reversa (LR) (Política Nacional de Resíduos Sólidos - PNRS, 2010).

A LR é conceituada como "o processo de planejamento, implementação e controle da eficiência e custo efetivo do fluxo de matérias-primas, estoques em processo, produtos acabados e as informações correspondentes do ponto de consumo ao ponto de origem, com a finalidade de recapturar valor ou eliminação adequada" (ROGERS; TIBBEN-LEMBKE, 1998, p. 2).

No Brasil, a PNRS versa sobre a responsabilidade compartilhada pelo ciclo de vida dos produtos. Segundo a lei 12.305 de 2010, a responsabilidade compartilhada é o total de deveres individuais e coletivos dos fabricantes, importadores, comerciantes, distribuidores, consumidores e poder público, a fim de reduzir o volume de resíduos sólidos e rejeitos gerados, assim como minimizar os impactos que afetam a saúde humana e a qualidade do meio-ambiente resultantes do ciclo de vida dos produtos.

Pela PNRS, todos os stakeholders da LR possuem responsabilidades conjuntas para viabilizar a recuperação de produtos. Um dos importantes stakeholders neste processo são as organizações intermediárias entre o usuário final e a empresa proprietária dos produtos. Na literatura 
consolidada de LR, esses agentes, também conhecidos como terciários - third party reverse logistics providers (3PRLPs), desempenham funções que muitas vezes não são possíveis para os produtores devido às condições econômicas (MEYER, 1999) ou falta de conhecimento técnico (KRUMWIEDE; SHEU, 2002).

Devido aos terciários apresentarem um papel relevante na LR, esse estudo foi desenvolvido para identificar as responsabilidades desses distribuidores ao longo de uma rede de fluxo reverso em um modelo de negócio PSS. Para isso, selecionou-se o setor de equipamentos eletromédicos, em especial a LR de bombas de infusão, da qual pertencem os equipamentos eletromédicos (PEREIRA; SILVEIRA, 2014), visto que a indústria eletroeletrônica, vem sofrendo pressões legislativas para adotar estruturas de LR (e.g. PNRS de 2010).

O artigo está estruturado da seguinte forma: após a seção de introdução, a seção 2 apresenta a revisão na literatura dos temas que norteiam a pesquisa; a seção 3 contém todos os métodos de pesquisa utilizados para o desenvolvimento do estudo; a seção 4 apresenta as resultados obtidos; e, por fim, as conclusões constam na seção 5.

\section{REVISÃO TEÓRICA}

\subsection{Panorama sobre Sistemas Produto-Serviço e Logística Reversa}

Um PSS pode trazer benefícios para diversos envolvidos. Tal modelo de negócio melhora o potencial de inovação e aumenta a competitividade dos provedores (BEUREN; FERREIRA; MIGUEL, 2013). Para empresas manufatureiras de produtos, a adição de serviços permite criação de maior valor para os clientes (BAINES et al., 2007), além da melhoria do relacionamento direto com os clientes devido ao fluxo de informações sobre suas preferências (GOEDKOOP et al., 1999; MONT, 2002).

Para os consumidores, este sistema integrado diminui as responsabilidades durante o uso do produto, como serviços de instalação e manutenção (BEUREN; FERREIRA; MIGUEL, 2013) e, em muitos casos, o usuário não fica responsável pelo descarte do produto ao término de sua vida útil (BAINES et al., 2007).

Entretanto, há barreiras que podem impedir a implementação ou aceitação de PSSs. Pode ser que a empresa não consiga aderir o sistema integrado devido à carga dos investimentos iniciais 
e despesas operacionais (CHIERICI; COPANI, 2016). Outra barreira, de fator organizacional, são infraestruturas de logística necessárias para a concretização do oferecimento dos serviços e gerenciamento dos bens após seus ciclos de vida (CHIERICI; COPANI, 2016).

Superar tais barreiras contribui com o meio-ambiente, pois o PSS é visto também como uma abordagem em direção à sustentabilidade (UNEP, 2002). Devido à oferta de serviços, é possível minimizar o total de material consumido (BEUREN; FERREIRA; MIGUEL, 2013), contribuindo para um uso mais eficaz de recursos (GOEDKOOP et al., 1999).

Além disso, práticas mais sustentáveis, como reuso e remanufatura, focam no eficiente uso do recurso ao longo de seu ciclo de vida e não na venda intensiva do produto (CHIERICI; COPANI, 2016). Tais estratégias têm o potencial de maximizar a vida útil do produto e aumentar a rentabilidade do negócio (CHIERICI; COPANI, 2016), favorecendo também a ideia de se ter diversos ciclos de vida interconectados de um mesmo produto (UNEP, 2002).

Porém, para a operacionalização de ciclos contínuos de uso de um artefato, há a necessidade de se estabelecer estruturas de LR. Para Brito e Dekker (2003), as etapas da logística reversa de um produto ordenam-se com a coleta em primeiro lugar, seguido pela inspeção, seleção, triagem, recuperação do produto e, por fim, redistribuição. Tal processo pode gerar ganhos diretos às fornecedoras por diminuir custos de descarte e de consumo de matéria-prima, sendo uma ferramenta do desenvolvimento sustentável (BRITO; DEKKER, 2003).

A LR em PSS contribui na gestão de ativos e no suporte à realização de serviços (CHIERICI; COPANI, 2016). Além disso, ela é tida como um instrumento de desenvolvimento econômico e social (PNRS, 2010), que possibilita a criação de valor por meio de materiais e produtos usados e coletados, devolvidos posteriormente à indústria (ELLEN MACARTHUR FOUNDATION - EMF, 2013). Desta forma, a LR favorece o fortalecimento de um modelo circular, que desvincula o crescimento global do consumo de recursos limitados, migrando de um sistema linear de produção (extrair-transformar-descartar) para uma economia que busca a circularidade contínua de materiais (EMF, 2013).

\subsection{Os Intermediários na Logística Reversa}

A partir do momento que uma empresa entende a importância de uma estrutura de LR, de acordo com Meade e Sarkis (2002) há três opções de escolha concernentes ao desenvolvimento da 
mesma: não adotar nenhuma medida, desenvolver um rede de LR própria, ou trabalhar em conjunto com um provedor terceirizado. Porém, adotar a LR tem sido algo desafiador, pois sua implementação demanda tempo, esforço e recursos financeiros (MEYER, 1999). Além disto, muitas empresas entendem que esta não é uma área tão importante em relação à outras questões da organização (ROGERS; TIBBEN-LEMBKE, 1998).

Devido a estes fatores, em conjunto com a falta de conhecimento sobre LR (KRUMWIEDE; SHEU, 2002), fabricantes de produtos são incapazes de ou não desejam assumir esta responsabilidade, necessitando, assim, de provedores terciários (3PRLPs) (COTTRILL, 2000; KRUMWIEDE; SHEU, 2002). Tais provedores podem ser caracterizados como intermediários independentes, envolvidos em atividades como coleta e processamento (BRITO; DEKKER, 2003).

Benefícios podem ser alcançados com a incorporação de tais provedores graças à existência de centros de retorno centralizados (CRCs), favorecendo uma melhoria no serviço ao consumidor (MEYER, 1999), aumentando a satisfação deste devido à rapidez na redistribuição dos produtos (MEADE; SARKIS, 2002). Além disso, com a contratação de 3PLRPs, alivia-se a carga das empresas fornecedoras da oferta, pois as permite focar em atividades relacionadas ao seu core empresarial (GIRI; SARKER, 2017).

Sahay e Mohan (2003) afirmam que as principais razões para fazer uso de tais provedores são: redução de custo, razões estratégicas, eficácia de processos e falta de capacidade interna. Além disso, a participação destes vem aumentando, desempenhando papel crescente na gestão integrada da cadeia de suprimento, gerando considerável impacto na performance total das empresas (GOVINDAN et al., 2012).

\section{METODOLOGIA}

Para obtenção dos dados foram realizadas entrevistas semiestruturadas com quatro empresas que prestam serviços de manutenção preventiva e corretiva de bombas de infusão. Essas empresas são representantes dos fabricantes de equipamentos médicos, e são responsáveis pela completude do PSS.

Nessa pesquisa, as empresas selecionadas foram denominadas por A, B, C e D por questões de confidencialidade. Para a coleta de dados, foi elaborado um questionário contendo perguntas 
sobre contratos com os clientes, treinamento operacional, manutenções, comunicação, coleta do equipamento e aspectos positivos e negativos do modelo de negócio.

Com as empresas A, B e C, o questionário foi aplicado através de entrevistas por telefone conduzidas pelos autores. Com a empresa D, por questões de política interna, foi possível apenas que as perguntas fossem respondidas por e-mail. Informações mais detalhadas sobre cada organização encontram-se na Tabela 1.

TABELA 1 - Informações sobre as empresas participantes

\begin{tabular}{lccc}
\hline Empresa & Localização & Função do & Tempo de \\
& & Entrevistado & Experiência \\
\hline Empresa A & Ribeirão Preto-SP & Gerente Comercial & 5 anos \\
\hline Empresa B & Campinas - SP & Diretor Comercial & 25 anos \\
\hline Empresa C & Sorocaba - SP & Estagiánia em Vendas & 1 ano \\
\cline { 2 - 4 } & & Intemas & 10 anos \\
\hline Empresa D & Belo Horizonte-MG & Diretor & 30 anos \\
\hline
\end{tabular}

As entrevistas realizadas por telefone foram inteiramente gravadas e então foi feita uma transcrição de cada uma delas. O questionário respondido por e-mail foi agrupado às demais entrevistas.

\section{RESULTADOS E DISCUSSÃO}

\subsection{Descrição das organizações de estudo}

A empresa A mantém-se no ramo de distribuição de equipamentos eletromédicos há 19 anos, atuando na prestação de serviços para cerca de 80 clientes, situados em um raio de $200 \mathrm{~km}$. A empresa B está atuante neste setor há mais de 20 anos, possuindo cerca de 90 clientes, situados em uma proximidade de até $80 \mathrm{~km}$ de seu estabelecimento.

A terceira empresa (C), fundada em 1990, presta serviços associados ao produto bombas de infusão em uma área de mais de $100 \mathrm{~km}$ de raio, atendendo 67 clientes. Por fim, a empresa D 
está no mercado de equipamentos hospitalares desde 2009, atendendo clientes situados no mesmo estado federativo.

\subsection{Descrição do PSS da bomba de infusão}

O PSS do equipamento eletromédico bomba de infusão refere-se a concessão de equipamentos, por parte da fabricante, sem de fato a transferência da posse do mesmo para os clientes, como hospitais e clínicas médicas. Esse modelo de negócio é comumente denominado regime de comodato.

Neste sistema, é oferecido aos clientes serviços de calibração com manutenção das bombas, tanto preventivas como corretivas, além da realização da entrega dos equipamentos no início do contrato e de coleta ao término do mesmo. Quando do seu término, as bombas de infusão passam por um processo de recondicionamento, a fim de que estejam em condições favoráveis para que, em seguida, sejam destinadas a outros clientes.

Os clientes são isentos de quaisquer custos relacionados a estes serviços, tendo a única obrigação de comprar os equipos em quantidade pré-estabelecida (em geral 10 equipos por bomba de infusão a cada mês). Tais equipos são desenvolvidos para serem acoplados apenas em equipamentos da própria fabricante, sendo a venda destes dispositivos a fonte de receita primária da fornecedora do PSS.

\subsection{Responsabilidades dos distribuidores em uma rede LR}

Inicialmente, é necessário que haja um contrato firmado entre a fornecedora do PSS e o cliente. Para que isto ocorra, os distribuidores possuem função importante, dialogando diretamente com o cliente, responsabilizando-se pela tratativa de assinatura. Porém, a formulação das cláusulas, verificação e emissão do contrato é de responsabilidade do provedor do PSS.

Após firmar-se oficialmente o acordo, os equipamentos são encaminhados ao cliente. $\mathrm{Na}$ maioria dos casos, a provedora do PSS é responsável por esse transporte, podendo contratar uma transportadora para tal serviço. Porém, a empresa $\mathrm{C}$ afirma que, se o cliente localizar-se perto de seu centro de distribuição, ela pode fazer a entrega.

Um dos serviços prestados pelas distribuidoras é a realização de treinamentos sobre o uso adequado do produto, sendo coordenados por equipes de técnicos e enfermeiros capacitados. 
Estes treinamentos são dados logo no início do contrato, após o recebimentos das bombas de infusão. Dependendo da quantidade de funcionários do cliente e turnos de trabalho, demora-se até quatro dias consecutivos até que todos sejam treinados.

Os distribuidores também são responsáveis por serviços técnicos, como manutenção preventiva e corretiva dos equipamentos. Tais procedimentos geralmente são realizados dentro do estabelecimento das distribuidoras, devido ao fato de terem um ambiente preparado e próprio para isto, com todo aparato técnico e ferramental.

Nesse caso, a LR dos equipamentos para o centro de distribuição é realizada, em grande parte das vezes, pelas próprias distribuidoras, podendo ser que o cliente faça essa atividade. Em caso de necessidade de novas bombas ou peças de reposição para o equipamento, a fabricante envia a mercadoria através de uma transportadora para as empresas A e D. No caso da empresa C, a própria distribuidora se encarrega de fazer a retirada das peças na fabricante, e em geral, a empresa B também faz esta retirada, porém dependendo da quantidade de mercadoria a fabricante se responsabiliza pelo envio. Durante o período de manutenção, as distribuidoras assumem todos custos de manutenção e de transporte de mercadorias destinadas a elas, enquanto a fabricante arca com os custos das peças e de transporte de alguma mercadoria destinada diretamente ao cliente como, por exemplo, a primeira entrega do equipamento.

Para que o cliente não fique sem bombas disponíveis durante o período de manutenção, o mesmo pode contratar um número maior de equipamentos do que o necessário, para que algumas sirvam de reservas. Porém nem sempre isto acontece, e nesse caso as empresas A e B afirmam deixar à disposição do cliente outras bombas enquanto as dele estão em manutenção.

Diferentemente, para a empresa $\mathrm{C}$, quando o cliente coloca em funcionamento todos os equipamentos, a distribuidora não consegue fazer a manutenção de todos de uma só vez, pois não há a disponibilização de bombas de infusão reservas. Neste caso ela realiza um sistema de rodízio de manutenção, que consiste na manutenção de um número parcial de equipamentos realizado sucessivamente, até que todas as bombas de infusão passem pela manutenção necessária. Já a empresa D apenas informou que não oferece equipamentos reservas. 
O tempo consumido desde a coleta dos equipamentos até a devolução ao cliente não é fixo, sendo dependente da intensidade do fluxo de equipamentos encaminhados para manutenção e da necessidade ou não da solicitação de novas peças, variando de 48 horas a 15 dias.

Em caso da constatação de peças danificadas ou defeituosas, as distribuidoras se responsabilizam pela LR ao encaminhá-las para a fabricante, sendo que esta é responsável pelo descarte adequado destes itens. Após realizadas as manutenções necessárias, as distribuidoras redistribuem os equipamentos aos clientes.

Durante o período contratual, a comunicação dos distribuidores se mantém tanto com a fabricante quanto com os clientes. Para ambos o canal mais utilizado é o $e$-mail, podendo ser também por outro aplicativo online ou por telefone, sendo importantes instrumentos para a LR, pois através deles os clientes relatam problemas detectados nos equipamentos, informando a necessidade de coleta dos mesmos.

Todas as empresas participantes afirmaram realizar rastreamento dos equipamentos distribuídos, vinculando a nota fiscal com o número de série do equipamento e com os dados jurídicos do cliente. Possuem também controle de equipamentos que precisam ser calibrados e histórico de manutenções em um sistema próprio.

Ao encerrar o contrato do PSS com o cliente, é necessário o retorno dos equipamentos. As empresas B e C se responsabilizam por esse transporte reverso, dos clientes até a fabricante. A empresa A afirma que a própria fabricante realiza esta atividade, e a empresa D é responsável pelo transporte até seu centro de distribuição seguido pela coleta dos equipamentos por parte da fabricante, que, por sua vez, realiza o recondicionamento deles. Em resumo, a Figura 1 apresenta as responsabilidades dos distribuidores associadas a LR das bombas de infusão. 


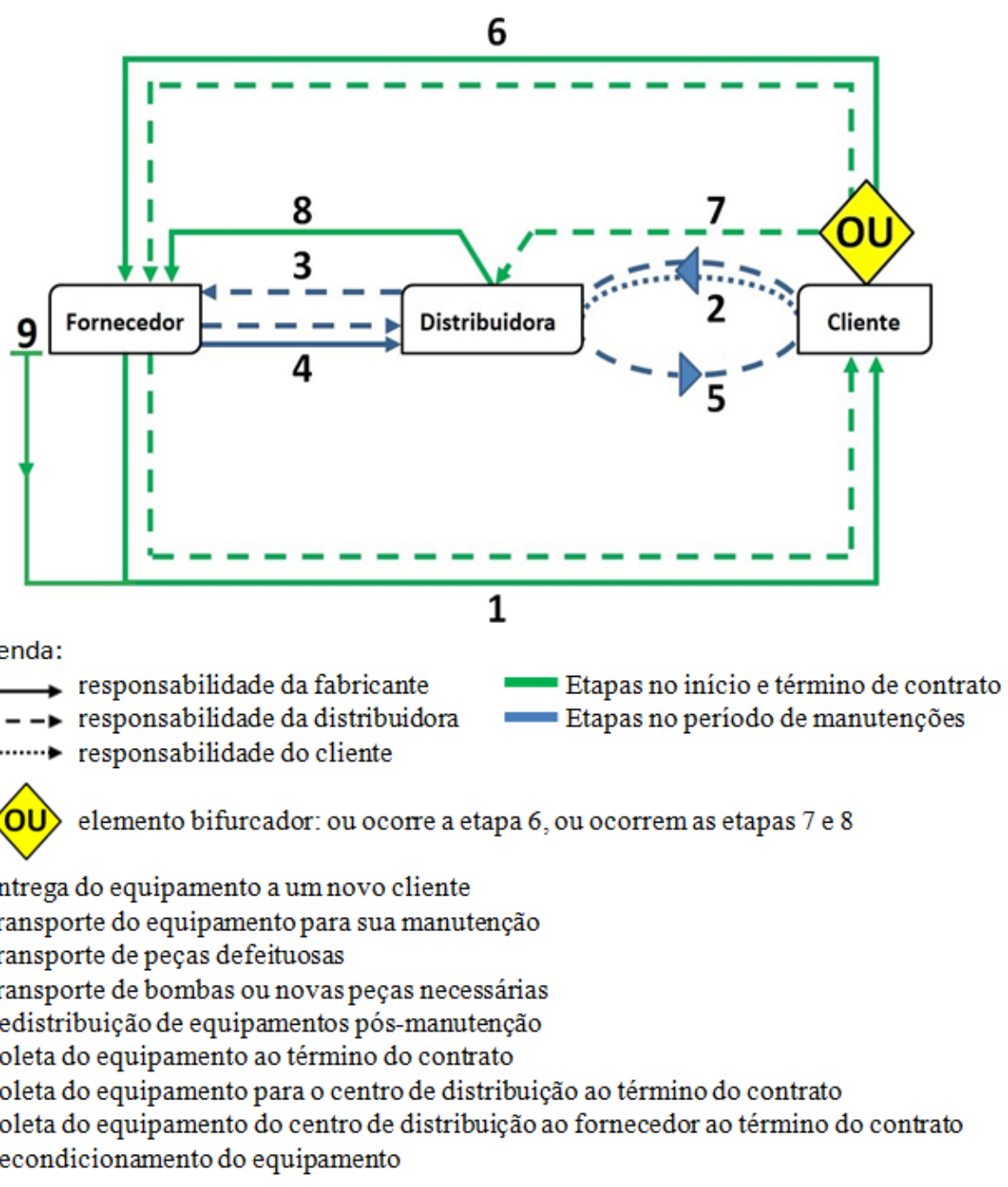

FIGURA 1 - Responsabilidades dos terciários na rede de LR. Fonte: Autores (2019)

\subsection{Vantagens e desvantagens associadas à LR e ao PSS}

De modo geral, as empresas A, B e C estão satisfeitas com o papel que exercem como terciários e com os resultados angariados dentro do PSS de bombas de infusão. A empresa A ressalta que é vantajoso para a fornecedora e para a distribuidora trabalhar com o comodato, pois, neste modelo, com a alta comercialização de equipos, obtém-se o ressarcimento dos equipamentos. A empresa B reconhece que o modelo de negócio é bem estruturado e que a política de pósvenda da fabricante atende muito bem ao cliente.

A empresa $\mathrm{C}$ destaca que este comodato fomenta uma relação duradoura com os clientes, sendo também financeiramente atrativo. Além disso, entende-se que seja melhor realizar as manutenções no seu centro de distribuição, pois são serviços que demandam muito tempo. Ao 
concentrar-se os equipamentos em um só lugar, favorece-se um fluxo maior de bombas de infusão circulando neste processo, não necessitando de diversas equipes técnicas se deslocando para os diversos clientes. Em contrapartida, a empresa B considera que realizar manutenções no cliente seria mais benéfico, pois não precisaria coletar e redistribuir posteriormente os equipamentos.

Para a empresa A, a maior dificuldade para realização da LR dos equipamentos é a grande distância a que certos clientes estão do seu centro de distribuição. Para a empresa C, um problema detectado é o fato de os clientes geralmente colocarem todos os equipamentos em funcionamento simultaneamente, o que dificulta a coleta dos mesmos para a manutenção. Concomitantemente, um grande desafio é a devolução dos equipamentos ao cliente dentro do menor tempo possível, sem deixá-lo insatisfeito.

Já para a empresa $\mathrm{D}$, só se tem vantagens em trabalhar neste sistema de comodato se houverem grandes quantidades de equipamentos envolvidos, sendo, em geral, não satisfatório para a distribuidora. Para ela, a má organização de clientes é um fator dificultador na coleta de bombas para manutenção e consequente prestação de serviços, enquanto também lida com falta de pagamentos por parte da fornecedora pelas manutenções realizadas.

\section{CONCLUSÃO}

Esse estudo analisou as responsabilidades de quatro terciários associados à LR do equipamento médico bomba de infusão. Nota-se que neste modelo de negócio, os intermediários possuem um papel importante para que a oferta produto-serviço seja usufruída pelos clientes. Como visto na Figura 1, os distribuidores se envolvem em quase todas as etapas de transporte do equipamento, inclusive nas de fluxo reverso, propiciando a realização de manutenções e recondicionamento.

O modelo de negócio analisado vai ao encontro de ideias apresentadas na literatura (e.g Beuren, Ferreira e Miguel (2013); Giri e Sarker (2017); UNEP (2002)), visto que o PSS de bombas de infusão, associado a uma rede de LR apoiada por intermediários, promove: maior inovação e competitividade para o fornecedor da oferta assim como redução de suas obrigações (como transportes e manutenções); benefícios financeiros para os clientes e redução de suas 
responsabilidades; múltiplos ciclos de vida do equipamento com a criação de valor por meio de produtos usados, reduzindo o uso intensivo de recursos.

Desta forma, observa-se que algumas responsabilidades concernentes aos distribuidores são relevantes para modelos de negócio vinculados à economia circular, pois permitem que produtos sejam reutilizados por novos consumidores, fomentando o uso sequencial destes e a diminuição do descarte e desperdício.

Além disso, os intermediários exercem outras atividades neste modelo de negócio, como treinamentos in loco nos hospitais e captação de novos clientes. Desta forma, destaca-se a importância da gestão destes terciários por parte do fornecedor do PSS, pois eles exercem funções que vão além do escopo da LR, sendo fundamentais para o sucesso da entrega da solução. Pesquisas futuras poderiam fazer uso de análise quantitativa de resultados, que podem ser estudadas em conjunto com as satisfações e insatisfações das empresas, buscando entender quais responsabilidades poderiam ser alteradas para um melhor funcionamento do sistema.

\section{AGRADECIMENTOS}

Agradecemos ao Programa Unificado de Bolsas de Estudo para Apoio e Formação de Estudantes de Graduação (PUB-USP) e à Fundação de Amparo à Pesquisa do Estado de São Paulo (FAPESP) pelo suporte financeiro, sob o processo $n^{\circ}$ 2017/06144-5. As opiniões, hipóteses, conclusões ou recomendações expressadas neste artigo são de responsabilidades dos autores e não refletem necessariamente as perspectivas da FAPESP e USP. Agradecemos também às empresas participantes da pesquisa que contribuíram com as informações levantadas.

\section{REFERÊNCIAS}

BAINES, T. S. et al. State-of-the-art in product service-systems. Proceedings of the Institution of Mechanical Engineers -- part b -- engineering manufacture, v. 221, p. 1543-1552, 2007.

BEUREN, F. H.; FERREIRA, M. G. G.; MIGUEL, P. A. C. Product-service systems: a literature review on integrated products and services. Journal of Cleaner Production, v. 47, p. 222-231, 2013.

BRASIL. Lei ${ }^{\circ}$ 12.305, de 2 de agosto de 2010. Institui a política nacional de resíduos sólidos; altera a lei no 9.605, de 12 de fevereiro de 1998; e dá outras providências. Diário Oficial da União, Brasília, DF, 3 ago. 2010. BRITO, M. P.; DEKKER, R. A Framework for reverse logistics. Roterdam: Erasmus Research Institute of 
Management, 2003. (ERIM Report Series Research in Management - ERS-2003-045-LIS).t, 2003.

COTTRILL, K. Return to sender. Traffic World, v. 262, n. 7, p. 17-18, 2000.

CHIERICI, E.; COPANI, G. Remanufacturing with upgrade PSS for new sustainable business models. Procedia CIRP, v. 47, p. 531-536, 2016.

ELLEN MACARTHUR FOUNDATION. Towards the circular economy: economic and business rationale for an accelerated transition. 2013. (596/09-08-2013).

GIRI, B. C.; SARKER, B. R. Improving performance by coordinating a supply chain with third party logistics outsourcing under production disruption. Computers \& Industrial Engineering, v. 103, p. 168-177, 2017.

GOEDKOOP, M. J. et al. Product service systems, Ecological and Economic Basics. [S.1.]: Product Innovation Technology Management, 1999.

GOVINDAN, K. et al. Analysis of third party reverse logistics provider using interpretive structural modeling. International Journal of Production Economics, v. 140, p. 204-211, 2012.

KRUMWIEDE, D. W.; SHEU, C. A model for reverse logistics entry by third-party providers. Omega, v. 30, p. 325-333, 2002.

MEADE, L.; SARKIS, J. A conceptual model for selecting and evaluating third-party reverse logistics providers. Supply Chain Management: An Internation Journal, v. 7, n. 5, p. 283-295, 2002.

MEYER, H. Many happy returns. Journal of Business Strategy, v. 20, n. 4, p. 27-31, 1999.

MONT, O. K. Clarifying the concept of product-service system. Journal of Cleaner Production, v. 10, p. $237-$ $245,2002$.

PEREIRA, M.; SILVEIRA, M. A. A Necessidade de adaptação às regulações ambientais da política nacional de resíduos sólidos: do fabricante ao consumidor organizacional no setor de equipamentos eletromédicos. Revista de Administração e Inovação - RAI, v. 11, n. 1809-2039, p. 88-109, 2014.

ROGERS, D. S.; TIBBEN-LEMBKE, R. S. Going backwards: reverse logistics trends and practices. Pittsburg: Reverse Logistics Executive Council, 1998.

SAHAY, B. S.; MOHAN, R. Supply chain management practices in Indian industry. International Journal of Physical Distribution and Logistics Management, v. 33, n. 7, p. 582-606, 2003.

SUNDIN, E.; BRAS, B. Making functional sales environmentally and economically beneficial through product remanufacturing. Journal of Cleaner Production, v. 13, p. 913-925, 2005.

UNITED NATIONS ENVIRONMENT PROGRAMME. Product-service systems and sustainability. Paris: UNEP, Division of Technology Industry and Economics, Production and Consumption Branch, 2002.

VEZZOLI, C. et al. New design challenges to widely implement "sustainable product-service systems". Journal of Cleaner Production, v. 97, p. 1-12, 2015. 